\title{
NUMERI CARATTERISTICI DEI FLOCCULI D'IDROGENO E DI CALCIO E DEI FILAMENTI D'IDROGENO PER GLI ANNI 1950-51-52
}

\author{
G. GoDoLI
}

E gia stata fatta menzione in questa sede delle difficoltà che si incontrano nel misurare le aree coperte dai fenomeni cromosferici [flocculi d'idrogeno, flocculi di calcin, filamenti d'idrogeno] ( ${ }^{1}$ ).

Fu appunto questa difficoltà che spinse a stimare tali aree per mezzo dei numeri caratteristici.

Purtroppo però, come è già stato dettagliatamente descritto nella nota citata, la bella serie dei numeri caratteristici, iniziatasi nel 1917, fu interrotta nel 1944. Sino al dicembre del 1946 abbiamo i numeri caratteristici stimati a M. Wilson e sino al dicembre del 1949 quelli stimati ad Arcetri.

Le ragioni principali che determinarono l'abolizione dei numeri caratteristici furono due:

a) il numero caratteristico è una grandezza troppo grossolana e soggettiva;

b) è inutile continuare a seguire il ciclo dell'attività solare attraverso tutte le sue manifestazioni (macchie, protuberanze, flocculi, filamenti) dato che esso è, almeno nelle sue linee generali, univoco.

Entrambe queste affermazioni furono dimostrate inesatte dalle ricerche di questi ultimi anni.

a) Il confronto fra l'area ed i numeri caratteristici dei fenomeni cromosferici $\left({ }^{1,2}\right)$ ha dimostrato che i secondi seguono abbastanza bene le variazioni delle prime col tempo potendosi considerare funzione di queste. Qualora le stime siano fatte con dei modelli, l'esperienza ha dimostrato che lo scarto medio non supera 0.2 unità per $\mathrm{i}$ bassi numeri e 0.5 per gli altri.

b) Le moderne ricerche sui filamenti e sui flocculi $\left(^{3,4}\right)$ hanno dimostrato che i cicli di questi fenomeni non coincidono né fra di loro né col ciclo delle macchie, risultando i tre cicli sfasati.

Allo stato attuale sembrano quindi superati i motivi per $i$ quali fu decisa l'abolizione dei numeri caratteristici. 
D'altra parte, se si escludono le carte-planisfero edite dall'osservatorio di Meudon, che danno però solo l'aspetto e la posizione media dei fenomeni cromosferici durante una rotazione solare, e le misure di Kodaikanal, che danno le medie semestrali dell'area proiettata $\left({ }^{1}\right)$ coperta dai filamenti, non abbiamo alcun dato sui fenomeni cromosferici.

Per queste ragioni crediamo utile dare nelle tabelle che seguono i numeri caratteristici per i flocculi d'idrogeno e di calcio e per i filamenti d'idrogeno per gli anni 1950-51-52.

I numeri caratteristici sono stati stimati sugli spettroeliogrammi raccolti alla torre solare di Arcetri nel periodo 1950-51-52.

Il rilievo spettroeliografico è sempre stato fatto con l'immagine di $64 \mathrm{~mm}$.

Le caratteristiche strumentali e la tecnica delle osservazioni $\dot{e}$ stata descritta in altra sede $\left(^{\mathbf{5}}\right)$.

Sono state esaminate complessivamente 334 lastre in $H_{n}$ e 336 lastre in $K_{2,3}\left({ }^{6}\right)$.

Il contributo dei singoli osservatori alla raccolta di tale materiale è qui sotto indicato:

\begin{tabular}{|c|c|c|c|c|c|c|c|c|c|c|c|c|c|}
\hline \multirow{2}{*}{\multicolumn{7}{|c|}{ Osservatori }} & \multicolumn{3}{|c|}{$\mathbf{H}_{\alpha}$} & \multicolumn{3}{|c|}{$K_{3 \cdot 3}$} & \multirow{2}{*}{ Totale } \\
\hline & & & & & & & 1950 & 1951 & 1952 & $19 \overline{0} 0$ & $495]$ & 1452 & \\
\hline Ballario & . & - & & & & . & 50 & 56 & 88 & 46 & 55 & SS & 383 \\
\hline Colacevich & & & & & & . & 一 & 1 & - & - & 1 & - & 2 \\
\hline Fracastoro & & & & & & . & - & 1 & - & - & 2 & - & 3 \\
\hline Godoli & - & • & • & & 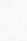 & - & 11 & - & 21 & 8 & - & 22 & 62 \\
\hline Hack . & - & - & - & & & . & - & 23 & - & - & 30 & - & 59 \\
\hline Righini & . & . & . & & & . & jó & 20 & 1 & 63 & 20 & - & 159 \\
\hline Rigutti & . & . & . & & & - . & 一 & - & 1 & - & - & 1 & I \\
\hline
\end{tabular}

La distribuzione mensile ed annuale delle lastre ridotte è data dai quadri seguenti :

\begin{tabular}{|r|r|r|r|r|r|r|r|r|r|r|r|r|r|}
\hline & 1 & 2 & 3 & 4 & 5 & 6 & 7 & 8 & 9 & 10 & 11 & 12 & Totale \\
\hline 1950 & 4 & 6 & 11 & 5 & 16 & 13 & 22 & 9 & 9 & 12 & 6 & 3 & 116 \\
51 & 9 & 3 & 5 & 8 & 11 & 12 & 15 & 15 & 9 & 7 & 6 & 7 & 107 \\
52 & 10 & 8 & 7 & 9 & 11 & 9 & 21 & 14 & 8 & 5 & 5 & 4 & 111 \\
\hline
\end{tabular}


$\mathrm{K}_{:, 3}$

\begin{tabular}{|r|r|r|r|r|r|r|r|r|r|r|r|r|r|}
\hline & 1 & 2 & 3 & 4 & 5 & 6 & 7 & 8 & 9 & 10 & 11 & 12 & Totale \\
\hline 1950 & 6 & 6 & 10 & 5 & 16 & 12 & 23 & 14 & 4 & 12 & 7 & 2 & 117 \\
51 & 8 & 4 & 6 & 8 & 10 & 13 & 15 & 15 & 11 & 8 & 5 & 5 & 108 \\
52 & 9 & 10 & 7 & 9 & 11 & 10 & 21 & 13 & 7 & 5 & 5 & 4 & 111 \\
\hline
\end{tabular}

La stima dei numeri caratteristici veniva eseguita con l'aiuto dei modelli di M. Wilson.

Per evidenti ragioni di uniformità, è stata stimata prima tutta la serie dei numeri caratteristici per i flocculi d'idrogeno; quindi tutta la serie per i flocculi di calcio ed infine tutta la serie per i filamenti d'idrogeno.

Come nelle stime eseguite in una nota precedente $\left({ }^{1}\right)$ lo scarto medio delle singole determinazioni non supera 0.2 unità per $\mathrm{i}$ bassi numeri e 0.5 per grli alti.

I risultati conseguiti sono stati raccolti nelle seguenti tre tabelle di immediata interpretazione.

Firenze - Osservatorio Astrofisico di Arcetri - Centro di astrofisica del C.N.R. - Febbraio 1952.

\section{RIASSUNTO}

Nella presente nota si mostra, alla luce delle recenti ricerche, l'opportunità di continuare a raccogliere le stime dei numeri curatteristici. Si danno $i$ numeri caralleristici dei flocculi d'idrogeno e di calcio e dei filamenti d'idrogeno per gli anni 1950-51-52, secondo le osservazioni eseguite alla torre solare di Arcetri.

\section{SUMMARY}

In the present paper it is shoun, in view of recent researches, the interest of continuing to collect the estimates of characteristic numbers. The characteristic numbers of floculae of hydrogen and of calcium and of the filaments of hydrogen for the years 1950-51-52 are given according to the observations carried out at the solar tou er of Arcetri. 


\section{BIBLIOGRAFIA}

(1) G. Righinı e G. Godolı: Sui numeri caratteristici dell'attività solare. Ann. Geof. 3, 501 (1950).

(2) G. Righini e G. Govoli: The physical meaning of the character figures of solar phenomena. Journal of Geophysical Research 55, 415 (1950).

(3) M. et M.me L. D'AzAmвuJA, Étude d'ensemble des protubérances solaires et de leur évolution. Ann. de Meudon 6, 7 (1948).

(4) G. Righini e G. Godoli, Ricerche statistiche sul ciclo dei flocculi. I. Correlazione fra l'attività delle macchie e quella dei flocculi. Mem. S. A. It. 22, 3 (1950). Arcetri Oss. e Mem. 67, 3 (1950).

(5) G. Righini e G. GodoLi, Riduzione del materiale spettroeliografico raccolto alla torre solare di Arcetri nel periodo 1932-1949. Mem. S. A. It. 21, 333 (1950). Arcetri Oss. e Mem. 66, 133 (1950).

(6) Handbuch der Astrophysik 4, 11l. Springer. Berlin (1929). 


\section{Tabella I-a}

Numeri caratteristici dei flocculi d'idrogeno per l'anno 1950.

\begin{tabular}{|c|c|c|c|c|c|c|c|c|c|c|c|c|}
\hline 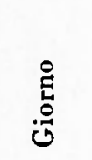 & 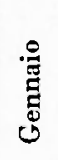 & 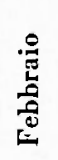 & $\stackrel{8}{\stackrel{\Sigma}{*}}$ & $\stackrel{0}{\stackrel{5}{L}}$ & 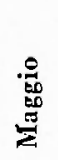 & 苞 & 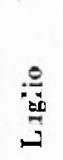 & 总 & 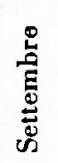 & 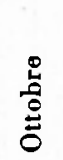 & $\begin{array}{l}\text { Lे } \\
\text { है } \\
\text { है } \\
\text { है }\end{array}$ & 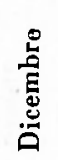 \\
\hline 1 & & & & & & & & & & & & \\
\hline $\begin{array}{l}1 \\
2\end{array}$ & - & - & - & - & 1.8 & - & 1.1 & - & - & - & - & 1.2 \\
\hline 3 & - & - & - & - & 1.8 & $-\overline{1}$ & $\overline{0 x}$ & - & $\overline{0}$ & - & - & - \\
\hline 4 & - & 10 & 1.1 & - & 1.7 & - & 0.9 & 1.3 & - & 0.3 & - & - \\
\hline 5 & - & - & - & 1.3 & 1.8 & 1.4 & - & 1.3 & 0.3 & 0.9 & - & - \\
\hline 6 & - & - & 1.4 & - & 1.2 & 1.5 & 1.3 & - & - & 0.8 & 1.0 & - \\
\hline 7 & - & - & 1.7 & 1.5 & - & 1.4 & 1.3 & 1.4 & 0.0 & - & - & - \\
\hline 8 & - & - & 1.7 & 1.6 & 1.4 & - & 1.2 & - & - & - & - & - \\
\hline$y$ & - & - & - & - & 1.5 & 1.6 & - & 1.3 & - & 1.0 & 0.8 & - \\
\hline 10 & - & - & - & - & - & 1.7 & 1.2 & 1.0 & - & - & 0.8 & - \\
\hline 11 & - & - & 1.8 & - & - & - & 1.2 & - & 0.1 & - & - & - \\
\hline 12 & 0.5 & - & - & - & 1.4 & & 1.2 & - & - & - & - & - \\
\hline 13 & & - & - & - & 1.1 & - & 1.2 & - & - & 0.8 & - & - \\
\hline 14 & 0.8 & - & - & - & - & - & 1.2 & - & - & 0.9 & $\cdots$ & - \\
\hline 15 & - & 1.1 & - & - & 1.1 & -. & - & - & - & $0 . y$ & - & - \\
\hline 16 & - & 2.0 & 1.9 & - & - & - & - & - & - & 0.9 & - & - \\
\hline 17 & - & 0.8 & 1.8 & - & 1.2 & - & 1.4 & - & - & 0.8 & - & - \\
\hline 18 & - & 1.8 & - & - & . & - & 1.4 & - & 0.5 & - & - & - \\
\hline 19 & - & - & - & - & - & - & 1.4 & - & - & - & - & - \\
\hline 20 & - & - & 0.8 & - & 1.2 & 1.2 & 1.5 & 1.6 & - & - & - & 0.0 \\
\hline 21 & - & - & - & - & - & 1.3 & 1.3 & 1.4 & - & - & 0.4 & 0.0 \\
\hline $2 \cdot 2$ & - & 1.1 & - & - & - & 1.3 & 1.3 & - & 0.4 & - & - & - \\
\hline 23 & - & - & 0.7 & -. & - & 1.3 & - & - & 0.4 & - & - & - \\
\hline 2.1 & - & - & 0.7 & - & 1.6 & - & - & - & - & - & - & - \\
\hline 25 & 2.0 & - & - & - & 1.5 & - & 1.4 & 1.3 & 0.6 & - & - & - \\
\hline 26 & - & - & - & - & - & - & 1.3 & - & - & - & - & - \\
\hline 27 & - & - & - & - & 1.6 & 1.4 & 1.2 & - & - & 0.5 & 0.6 & - \\
\hline 28 & - & - & 1.4 & - & - & 1.3 & 1.2 & - & - & - & 0.6 & - \\
\hline 29 & - & - & - & 1.6 & 1.3 & - & - & - & - & - & - & - \\
\hline 30 & 1.0 & - & - & 1.7 & - & 1.2 & - & 0.0 & 1.0 & 1.0 & - & $\cdots$ \\
\hline 31 & - & - & - & - & - & - & 1.1 & - & - & 0.9 & - & - \\
\hline Media & 1.1 & 1.3 & 1.4 & 1.5 & 1.4 & 1.4 & 1.2 & 1.2 & 0.5 & 0.8 & 0.7 & 0.4 \\
\hline \multicolumn{13}{|c|}{ Media annuale 1.14} \\
\hline
\end{tabular}




\section{Tabella I-],}

Numeri caratteristici dei flocculi d'idrogeno per l'anno 1951.

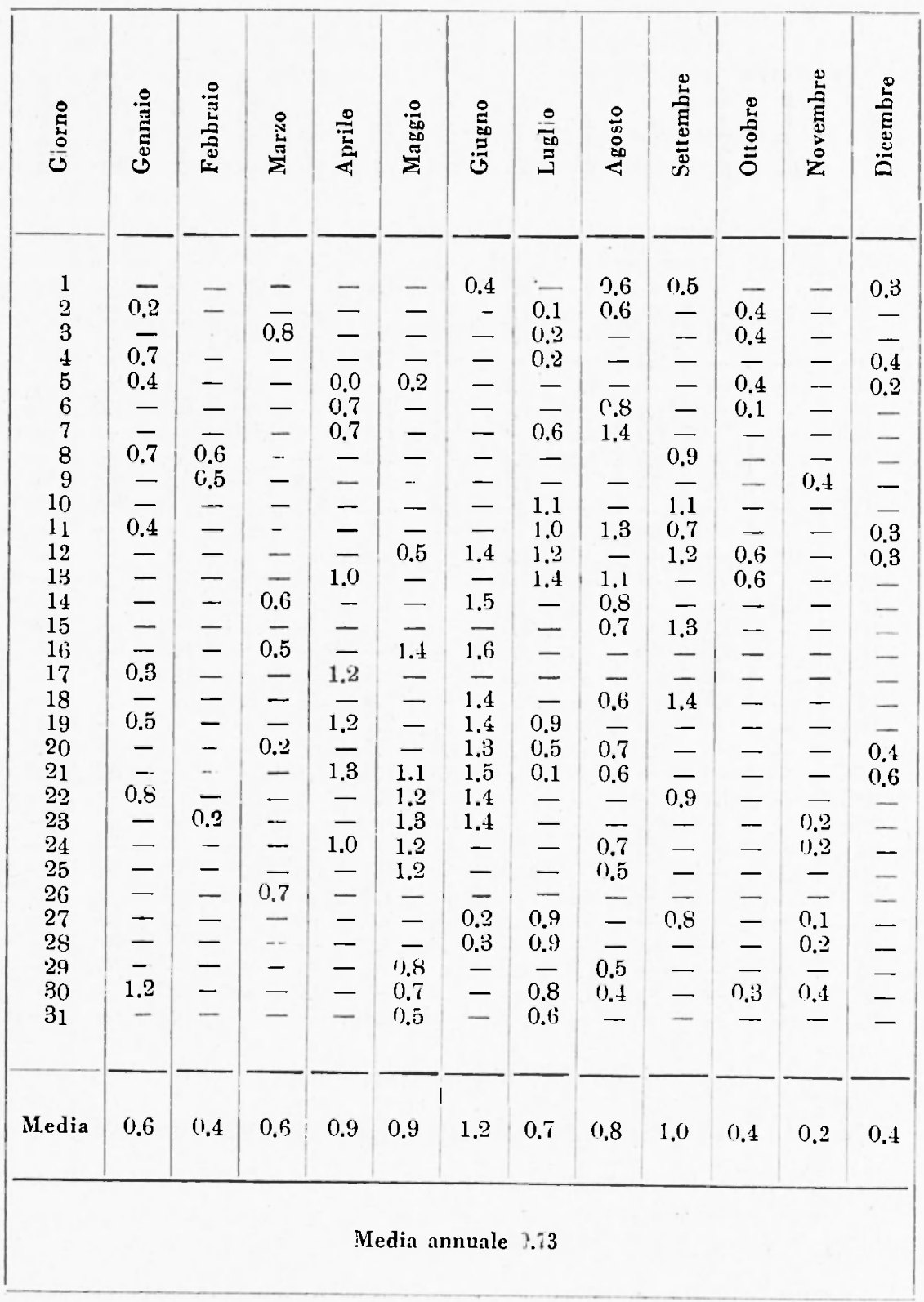


Tabella I-c

Numeri caratteristici dei flocculi d'idrogeno per l'anno 1952.

\begin{tabular}{|c|c|c|c|c|c|c|c|c|c|c|c|c|}
\hline $\begin{array}{l}\stackrel{\circ}{E} \\
\stackrel{9}{0}\end{array}$ & 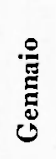 & 总 & 总 & $\stackrel{\stackrel{0}{\vec{L}}}{\stackrel{0}{2}}$ & 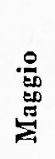 & 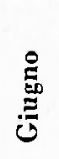 & 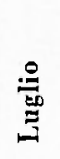 & 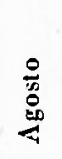 & 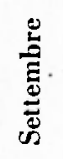 & $\frac{\mathscr{D}}{\stackrel{\Xi}{0}}$ & 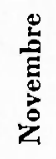 & \\
\hline 1 & & & & & & & & & & & & \\
\hline $\begin{array}{l}1 \\
2\end{array}$ & $\overline{0.5}$ & - & - & $\bar{z}$ & $\bar{z}$ & $\bar{z}$ & 0.6 & $\begin{array}{l}0.5 \\
0.4\end{array}$ & $\begin{array}{l}1.0 \\
1.0\end{array}$ & $\overline{0.0}$ & $\bar{z}$ & $\overline{-}$ \\
\hline 3 & - & - & - & - & - & 0.0 & 0.5 & - & 0.9 & - & - & - \\
\hline 4 & 0.5 & - & - & - & - & 0.0 & 0.4 & 0.4 & 0.8 & - & - & - \\
\hline 5 & 0.4 & - & - & - & 0.1 & 0.0 & 0.2 & 0.4 & - & - & - & - \\
\hline $\begin{array}{l}6 \\
7\end{array}$ & $\overline{04}$ & $\overline{0}$ & - & - & - & - & $\overline{0} 0$ & 0.5 & - & - & -- & - \\
\hline 8 & $\begin{array}{l}.4 \\
0.5\end{array}$ & 0.2 & $\overline{-}$ & $\overline{0 . \overline{2}}$ & $\overline{0.1}$ & - & $\frac{0.0}{-}$ & $\overline{-}$ & - & $\overline{-}$ & $\overline{0.2}$ & 二 \\
\hline 9 & - & 0.1 & - & 0.4 & 0.0 & 0.1 & - & 0.6 & 0.4 & 0.2 & - & - \\
\hline 10 & - & - & - & 0.3 & 0.0 & - & 0.2 & - & - & - & - & 0.0 \\
\hline 11 & - & - & 0.1 & - & -- & 0.0 & 0.2 & 0.5 & -- & - & - & - \\
\hline 12 & - & - & - & - & - & - & 0.3 & 0.3 & - & - & - & - \\
\hline 13 & - & - & - & - & 0.0 & 0.0 & - & 0.5 & - & - & 0.1 & - \\
\hline 14 & - & - & 0.2 & - & - & - & 0.4 & - & - & - & - & - \\
\hline 15 & 0.6 & - & 0.2 & 0.0 & 0.1 & - & 0.4 & - & - & - & 0.3 & - \\
\hline 16 & - & - & - & - & - & - & 0.5 & - & - & - & - & 0.3 \\
\hline 17 & - & - & - & 0.0 & - & 0.1 & 0.5 & - & - & 0.0 & - & - \\
\hline 18 & $\overline{0}$ & 0.3 & 0.1 & 0.2 & - & - & - & - & - & 0.0 & - & - \\
\hline 19 & 0.3 & 0.2 & - & 0.1 & - & - & 0.3 & - & - & - & - & 0.4 \\
\hline 20 & & 0.3 & - & - & - & - & - & —. & - & - & 0.3 & - \\
\hline 21 & 0.1 & - & 0.0 & - & 0.1 & - & 0.0 & - & - & - & 0.2 & - \\
\hline 22 & - & - & 0.0 & - & - & - & 0.0 & 0.4 & - & - & - & - \\
\hline 23 & - & - & - & - & 0.1 & - & 0.0 & - & 0.2 & - & - & 0.2 \\
\hline 24 & - & - & - & - & 0.1 & - & 0.0 & - & 0.3 & - & - & - \\
\hline 25 & - & 0.2 & - & - & - & - & & - & & - & - & - \\
\hline 26 & - & 0.0 & - & 0.1 & - & - & 0.0 & 0.4 & 0.3 & - & - & - \\
\hline 27 & & 0.0 & 0.1 & & - & - & - & 0,3 & - & - & - & - \\
\hline 28 & 0.2 & - & - & - & - & 0.3 & - & 0.5 & - & 0.3 & - & - \\
\hline 29 & 0.2 & - & - & 0.0 & - & -0 & 0.2 & 0.9 & - & - & - & - \\
\hline $\begin{array}{l}30 \\
31\end{array}$ & - & - & - & - & $\begin{array}{l}0.2 \\
0.1\end{array}$ & 0.2 & $\begin{array}{c}0.3 \\
0.4\end{array}$ & $\bar{z}$ & - & - & 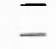 & $=$ \\
\hline Media & 0.4 & 0.2 & 0.1 & 0.1 & 0.1 & 0.1 & 0.3 & 0.5 & 0.6 & 0.1 & 0.2 & 0.2 \\
\hline \multicolumn{13}{|c|}{ Media } \\
\hline
\end{tabular}


TABELLA II-a

Numeri caratteristici dei flocculi di calcio per l'anno 1950.

\begin{tabular}{|c|c|c|c|c|c|c|c|c|c|c|c|c|}
\hline 芯 & 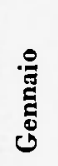 & 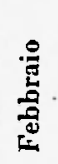 & 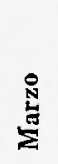 & $\stackrel{\vec{g}}{\frac{\pi}{4}}$ & 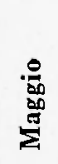 & 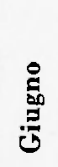 & 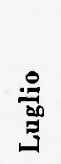 & 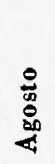 & & 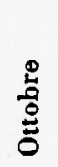 & 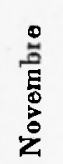 & 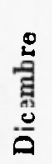 \\
\hline 1 & & & & & & & & & & & & \\
\hline 2 & - & - & - & - & $\begin{array}{l}2.6 \\
2.6\end{array}$ & - & 2.0 & - & - & - & - & 1.7 \\
\hline 3 & - & 2.4 & - & - & 2.7 & 2.5 & 1.8 & - & 1.7 & - & - & - \\
\hline 4 & - & - & - & - & - & - & 2.0 & 2.2 & - & 1.9 & - & - \\
\hline 5 & - & - & - & 3.0 & 2.6 & 2.4 & 1.8 & 2.2 & 1.5 & 2.0 & 2.0 & - \\
\hline 6 & - & - & 1.8 & - & 2.6 & 2.0 & 1.9 & - & - & 2.0 & 2.2 & - \\
\hline 7 & - & - & 3.2 & 2.8 & - & 1.8 & 2.2 & 2.2 & - & - & - & - \\
\hline 8 & - & - & 3.2 & 2.8 & 2.7 & - & 2.1 & 2.3 & - & - & - & - \\
\hline 9 & - & - & - & - & 2.6 & 1.7 & - & 2.3 & - & 2.8 & 2,1 & - \\
\hline 10 & - & - & - & - & - & 2.2 & 2.2 & 1.5 & - & - & 2.2 & - \\
\hline 11 & 2.4 & - & 3.4 & - & - & - & 2.3 & - & - & - & - & - \\
\hline 12 & 2.5 & - & - & - & 2.4 & - & 2.3 & - & - & - & - & - \\
\hline 13 & 2.2 & - & - & - & 2.8 & - & 2.4 & - & - & 2.0 & - & - \\
\hline 14 & 2.4 & - & - & - & - & - & - & - & - & 2.1 & - & - \\
\hline 15 & - & 2.8 & - & - & 2.6 & - & - & - & - & 2.1 & - & - \\
\hline 16 & - & 3.0 & 2.8 & - & - & - & - & - & - & 2.0 & - & - \\
\hline 17 & - & 3.2 & 2.8 & - & 2.2 & - & 2.0 & - & - & 2.0 & - & - \\
\hline 18 & - & 3.2 & - & - & - & - & 2.0 & - & 2.2 & - & - & - \\
\hline 19 & - & - & - & - & - & - & 2.4 & - & - & - & - & - \\
\hline 20 & - & - & 2.? & - & 2.4 & 2.6 & 2.3 & 2.2 & - & - & - & - \\
\hline 21 & - & - & - & - & - & 2.6 & 2.3 & 2.2 & - & - & 1.2 & 0.8 \\
\hline 22 & - & 2.6 & - & - & - & 2.7 & 2.8 & 2.4 & - & - & - & - \\
\hline 23 & - & - & 1.8 & - & - & - & - & 2.3 & 1.4 & - & - & - \\
\hline 21 & - & - & 2.0 & - & 2.9 & - & 2.6 & - & - & - & - & - \\
\hline 25 & 3.6 & - & - & - & 2.7 & - & 2.6 & 2.5 & - & - & - & - \\
\hline 26 & - & - & - & - & - & - & 2.5 & - & - & - & - & - \\
\hline 27 & - & - & - & - & 2.6 & 2.5 & 2.4 & 2.3 & - & 1.3 & 1.4 & - \\
\hline 28 & - & - & 2.4 & $=$ & - & 2.6 & 2.4 & 2.1 & - & - & 1.5 & - \\
\hline 29 & - & - & - & 2.7 & 2.4 & - & - & - & - & - & - & - \\
\hline 30 & 3.6 & - & - & 2.6 & - & 2.2 & - & 2.0 & - & 1.8 & - & - \\
\hline 31 & - & - & - & - & - & - & 2.1 & - & - & 3.0 & - & - \\
\hline Media & 2.8 & 2.9 & 2.6 & 2.8 & 2.6 & 2.3 & 2.2 & 2.2 & 1.7 & 2.0 & 1.8 & 1.2 \\
\hline \multicolumn{13}{|c|}{ Media annuale 2.30} \\
\hline
\end{tabular}




\section{TABELLA II-b}

Numeri caratteristici dei flocculi di calcio per l'anno 1951.

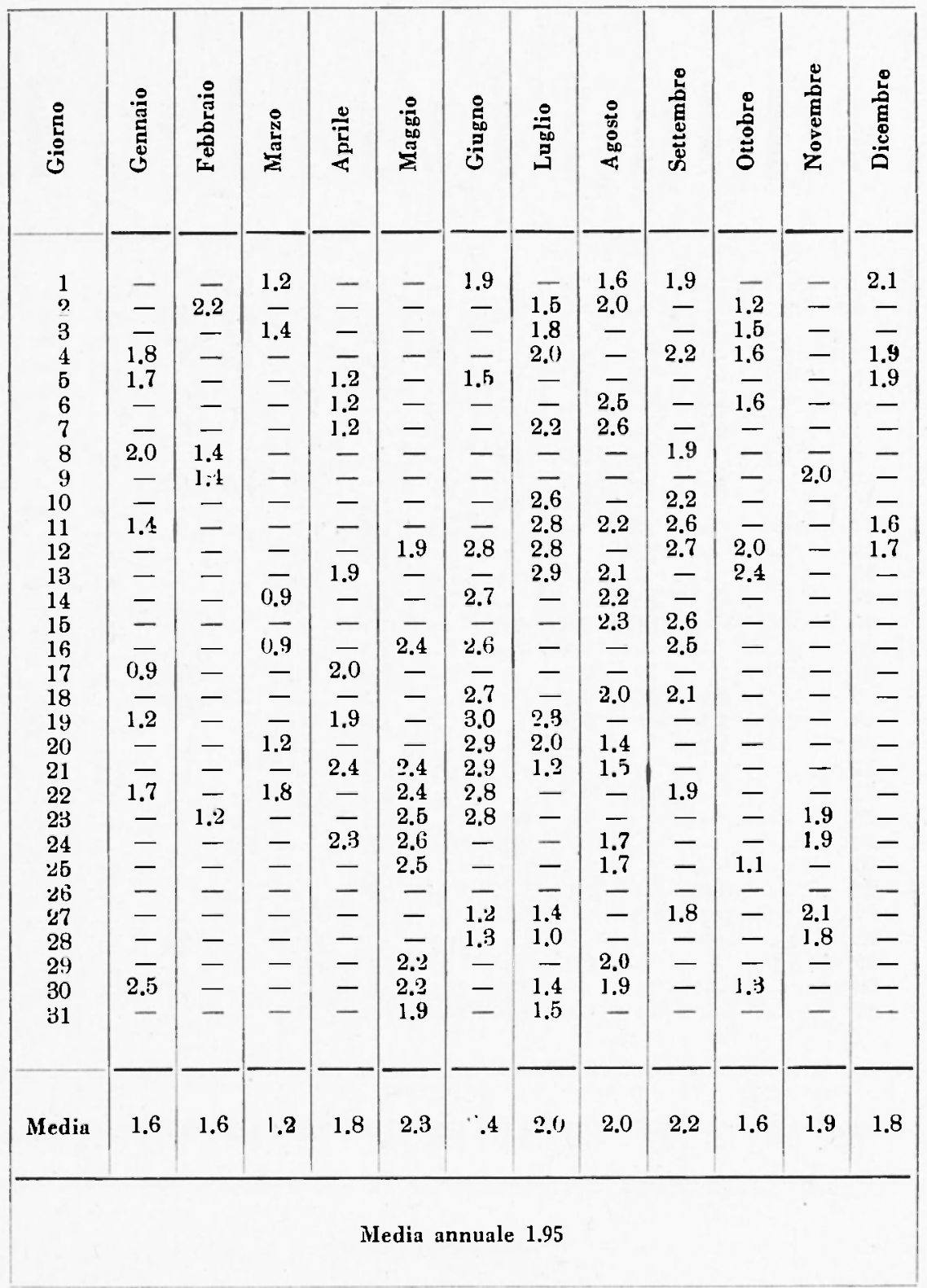




\section{TABella II-c}

Numeri caratteristici dei flocculi di calcio per l'anno 1952.

\begin{tabular}{|c|c|c|c|c|c|c|c|c|c|c|c|c|}
\hline 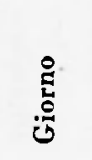 & 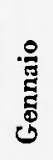 & 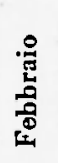 & 密 & $\begin{array}{l}\stackrel{g}{\vec{L}} \\
\text { 定 }\end{array}$ & 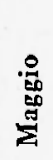 & 苋 & 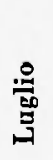 & 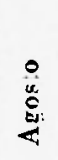 & 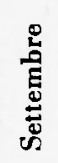 & 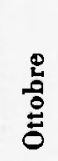 & 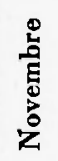 & 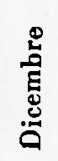 \\
\hline 1 & & & & & & & & & & & & \\
\hline 2 & $\overline{0}$ & - & - & - & - & - & 1.8 & 1.3 & 2.1 & - & - & - \\
\hline 3 & 2.1 & - & - & - & - & - & - & 1.1 & 2.0 & 0.4 & - & - \\
\hline 4 & $\overrightarrow{20}$ & - & - & - & - & 0.1 & 1.9 & - & 1.9 & - & - & - \\
\hline 5 & 2.0 & - & 二 & - & $\overline{10}$ & 0.2 & 1.0 & 1. & 1.8 & - & $\overline{7}$ & - \\
\hline 6 & - & 1.9 & - & - & 1.0 & 0.1 & 0.2 & 1.4 & $\overline{-}$ & I & 0.6 & 二 \\
\hline 7 & 0.9 & 2.1 & - & - & - & - & 0.0 & - & - & - & - & - \\
\hline 8 & 1.2 & - & - & 1.2 & 1.8 & - & - & - & - & - & 1.0 & - \\
\hline 9 & - & 1.7 & - & 1.3 & 1.6 & 0.0 & - & 1.8 & 1.3 & 0.8 & - & - \\
\hline 10 & - & $\rightarrow$ & - & 1.2 & 0.8 & - & 1.3 & - & - & - & - & 0.9 \\
\hline 11 & - & - & 0.3 & - & - & 0.4 & 1.6 & 1.7 & - & - & - & - \\
\hline 12 & - & - & 1.0 & - & - & - & 1.7 & 1.5 & - & - & - & - \\
\hline 13 & - & - & - & - & 0.4 & 1.0 & $=$ & 1.5 & - & - & 0.1 & - \\
\hline 14 & - & - & 1.2 & - & - & - & 1.7 & - & - & - & - & - \\
\hline 15 & 2.0 & - & 1.0 & 0.0 & 0.3 & - & 1.7 & - & - & - & 0.8 & - \\
\hline 16 & - & - & - & - & - & - & 1.9 & - & - & - & - & 1.8 \\
\hline 17 & - & - & - & 0.1 & - & 1.3 & 2.0 & - & - & 0.5 & - & - \\
\hline 18 & - & 1.2 & 1.0 & 0.8 & - & - & - & - & - & 0.2 & - & - \\
\hline 19 & 1.8 & 1.1 & - & 0.9 & - & - & 1.8 & - & - & - & - & 1.6 \\
\hline 20 & - & 1.1 & - & - & - & - & - & - & - & - & - & - \\
\hline 21 & - & - & - & - & 0.4 & 1.7 & 0.3 & - & - & - & 1.3 & - \\
\hline 22 & - & - & 0.0 & - & - & - & 0.3 & 1.3 & - & - & - & - \\
\hline 23 & - & - & - & - & 0.9 & - & 0.3 & - & 1.2 & - & - & 0.9 \\
\hline 24 & - & - & - & - & 1.3 & - & 0.6 & - & 1.2 & - & - & - \\
\hline 25 & - & 0.0 & - & - & - & - & - & - & - & - & - & - \\
\hline 26 & - & 0.0 & - & 1.1 & - & - & 0.6 & - & - & - & - & - \\
\hline 27 & - & 0.2 & 1.0 & - & - & - & - & 1.4 & - & - & - & - \\
\hline 28 & 1.4 & 0.0 & - & - & - & 2.0 & - & 1.8 & - & 1.4 & - & - \\
\hline 29 & 1.0 & - & - & 1.2 & - & - & 1.1 & 1.8 & - & - & - & - \\
\hline 30 & - & - & - & - & 1.2 & 2.1 & 1.0 & - & - & - & - & - \\
\hline 31 & - & - & - & - & 1.0 & - & 1.2 & - & - & - & - & - \\
\hline Media & 1.5 & 0.9 & 0.8 & 0.9 & 1.1 & 0.9 & 1.2 & 1.5 & 1.6 & 0.7 & 0.8 & 1.2 \\
\hline \multicolumn{13}{|c|}{ Media annuale 1.12} \\
\hline
\end{tabular}




\section{TABELLA III-a}

Numeri caratteristici dei filamenti d'idrogeno per l'anno 1950.

\begin{tabular}{|c|c|c|c|c|c|c|c|c|c|c|c|c|}
\hline 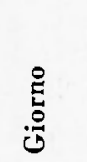 & Dُ & 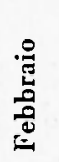 & $\underset{\mathbb{E}}{\stackrel{8}{\Sigma}}$ & 苞 & 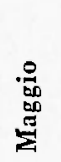 & 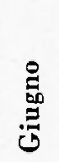 & 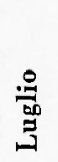 & 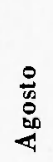 & 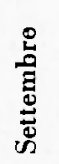 & 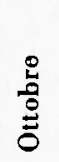 & 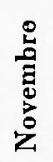 & 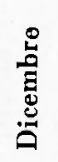 \\
\hline & & & & & & & & & & & & \\
\hline $\begin{array}{l}1 \\
2\end{array}$ & - & $\bar{E}$ & - & - & $\begin{array}{l}2.6 \\
27\end{array}$ & - & 2.2 & - & - & $\overline{-}$ & - & 1.2 \\
\hline 3 & - & 2.3 & - & - & 2.9 & 2.4 & 2.5 & - & 2.3 & - & - & - \\
\hline 4 & - & - & 1.6 & - & - & - & 2.6 & 2.8 & - & 1.8 & - & - \\
\hline 5 & - & - & - & 2.7 & 2.5 & 2.5 & - & 2.8 & 2.4 & 1.8 & - & - \\
\hline 6 & - & - & 2.2 & - & 2.7 & 2.6 & 1.9 & - & - & 1.9 & 1.4 & - \\
\hline 7 & - & - & 2.4 & 2.8 & - & 2.4 & 1.3 & 2.0 & 2.4 & - & - & - \\
\hline 8 & - & - & 2.0 & 2.9 & 2.6 & - & 1.8 & - & - & - & - & - \\
\hline 9 & - & - & - & - & 3.2 & 2.5 & - & 2,2 & - & 2.1 & 0.9 & - \\
\hline 10 & - & - & - & - & - & 2.4 & 1.7 & 2.0 & - & - & 1.0 & - \\
\hline 11 & - & - & 3.11 & - & - & - & 1.9 & - & 2.0 & - & - & - \\
\hline 12 & 0.8 & - & - & - & 3.0 & - & 2.0 & - & - & - & - & - \\
\hline 13 & - & - & -- & - & 3.2 & - & 1.9 & - & - & 1.7 & - & - \\
\hline 14 & 2.4 & - & - & - & - & - & 2.3 & - & - & 1.8 & - & - \\
\hline 15 & - & 2,6 & 一 & - & 3.0 & - & - & - & - & 1.6 & - & - \\
\hline 16 & - & 3.5 & 4.0 & - & - & - & - & - & - & 1.7 & - & - \\
\hline 17 & - & 3.4 & 4.3 & - & 2.9 & - & 2.9 & - & - & 1.7 & - & - \\
\hline 18 & - & 3.5 & - & - & - & - & 2.6 & - & 1.6 & - & - & - \\
\hline 19 & - & - & - & - & - & - & 2.8 & - & - & - & - & - \\
\hline 20 & - & - & 3.2 & - & 2.8 & 3.9 & 2.2 & 2.3 & - & - & - & 0.0 \\
\hline 21 & - & - & - & - & - & 2.4 & 1.9 & 2.0 & - & - & 0.3 & 0.4 \\
\hline $2 \overline{2}$ & - & 2.2 & - & - & - & 2.4 & 1.8 & - & 0.2 & - & - & - \\
\hline 23 & - & - & 3.0 & - & - & 2.1 & - & - & 0.2 & - & - & - \\
\hline 24 & - & - & 4.5 & - & 2.6 & - & - & - & - & - & - & - \\
\hline 25 & 3.0 & - & - & - & 2.2 & - & 2.8 & 0.9 & 0.2 & - & - & - \\
\hline 26 & - & - & - & - & - & - & 2.7 & - & - & - & - & - \\
\hline 27 & - & - & - & - & 2.6 & 3.0 & 2.6 & - & - & 2.3 & 1.0 & - \\
\hline 28 & - & - & 2.4 & $=$ & - & 2.9 & 2.7 & - & - & - & 1.0 & - \\
\hline 29 & - & - & - & 2.7 & 2.6 & - & - & $\overline{1}$ & - & $\overline{0}$ & - & E \\
\hline 30 & 4.0 & - & - & 2.6 & - & 2.2 & $=$ & 1.8 & 0.4 & 2.2 & - & - \\
\hline 31 & - & - & - & - & - & - & 2.7 & - & - & 2.3 & & \\
\hline Media & 2.6 & 2.9 & 3.0 & 2.7 & 2.8 & 2.6 & 2.3 & 2.0 & 1.3 & 1.9 & 0.9 & 0.5 \\
\hline
\end{tabular}

Media annuale 2.26 


\section{TABELLA III-b}

Numeri caratteristici dei filamenti d'idrogeno per lanno 1951.

\begin{tabular}{|c|c|c|c|c|c|c|c|c|c|c|c|c|}
\hline$\frac{\mathfrak{E}}{\frac{a}{u}}$ & 节 & 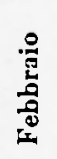 & 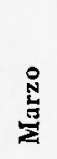 & 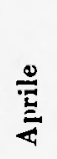 & 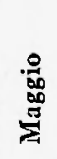 & 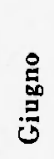 & $\begin{array}{l}\stackrel{3}{3} \\
\text { בై }\end{array}$ & 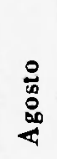 & 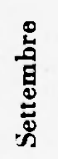 & 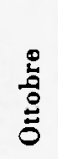 & 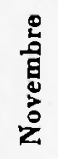 & 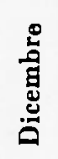 \\
\hline & & & & & & & & & & & & \\
\hline 1 & - & - & - & - & - & 0,1 & - & 0.6 & 1.5 & - & - & 1.5 \\
\hline 3 & 0.8 & - & - & - & - & - & 0,8 & 1.0 & - & 1.8 & - & - \\
\hline 3 & - & - & 2.6 & - & - & - & 1.0 & - & - & 1.4 & - & - \\
\hline 4 & 0.9 & - & - & - & - & - & 1.2 & - & - & - & - & 2.4 \\
\hline 5 & 1.1 & - & - & 0.2 & 0.4 & - & - & - & - & 0.9 & - & 2,2 \\
\hline 6 & - & - & - & 0.5 & - & - & - & 1.4 & - & 0.9 & - & $\therefore$ \\
\hline 7 & - & - & - & 0.6 & - & - & 2.7 & 1.3 & - & - & - & - \\
\hline 8 & 1.2 & 0.6 & - & - & - & - & - & - & 2.8 & - & - & - \\
\hline 9 & - & 1.0 & - & - & - & - & - & - & - & - & 0.7 & - \\
\hline 10 & - & - & - & - & - & - & 2.1 & - & 2.2 & - & - & - \\
\hline 11 & 1.3 & - & - & - & - & - & 2.1 & 1.4 & 2.1 & - & - & 1.6 \\
\hline 12 & - & - & - & - & 0.6 & 2.2 & 2.3 & $\rightarrow$ & 2.6 & 1.5 & - & 1.4 \\
\hline 13 & - & - & - & 0.3 & - & - & 2.4 & 1.4 & - & 1.7 & - & - \\
\hline 14 & - & - & 0.9 & - & - & 1.8 & - & 1.2 & - & - & - & - \\
\hline 15 & - & - & - & - & - & - & - & 1.1 & 2.6 & - & - & - \\
\hline 16 & - & - & 1.1 & - & 0.9 & 1.9 & - & - & - & - & - & - \\
\hline 17 & 0.3 & - & - & 1.0 & - & - & - & - & - & - & - & - \\
\hline 18 & - & - & - & - & - & 1.0 & - & 2.0 & 2.0 & - & - & - \\
\hline 19 & 0.1 & - & - & 1.0 & - & 0.6 & 1.6 & - & - & - & - & - \\
\hline 20 & - & - & 0.8 & - & - & 0.6 & 0.9 & 1.2 & - & - & - & 0.8 \\
\hline 21 & - & - & - & 11.5 & 0.1 & 0.6 & 0.9 & 0.8 & - & - & - & 0.9 \\
\hline 22 & 0.6 & - & - & - & 0.2 & 0.7 & - & - & 1.2 & - & - & - \\
\hline 23 & - & 1.3 & - & - & 0.3 & 0.8 & - & - & - & - & U.4 & - \\
\hline 24 & - & - & - & 0.6 & 0.2 & - & - & 0.9 & - & - & 0.6 & - \\
\hline 25 & - & - & - & - & 0.1 & - & - & 0.6 & - & - & - & - \\
\hline 26 & - & - & $2.1)$ & - & - & - & - & - & - & - & - & - \\
\hline 27 & - & - & - & - & - & 0.3 & 0.7 & - & 1.9 & - & 0.8 & - \\
\hline 28 & - & - & - & - & - & 0.4 & 0.8 & - & - & - & 1.2 & - \\
\hline 29 & $=$ & - & - & - & 0.4 & - & - & 1.2 & - & - & - & - \\
\hline 3) & 0.7 & - & - & - & 0.2 & - & 0.2 & 1.6 & - & 1.1 & 1.6 & - \\
\hline 31 & - & - & - & - & 0.6 & - & 0.4 & - & - & - & - & - \\
\hline Media & 0.8 & 1.0 & 1.5 & 0.6 & 0.4 & 0.9 & 1.3 & 1.2 & 2.1 & 1.3 & 0.9 & 1.5 \\
\hline \multicolumn{13}{|c|}{ Media annuale 1.11} \\
\hline
\end{tabular}




\section{TABELLA III-c}

Numeri caratteristici dei filamenti d'idrogeno per l'anno 1952.

\begin{tabular}{|c|c|c|c|c|c|c|c|c|c|c|c|c|}
\hline 芯 & 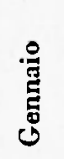 & 承 & 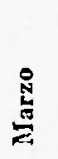 & 苞 & 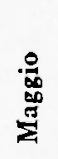 & 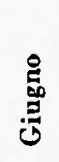 & 窇 & 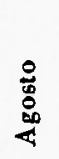 & 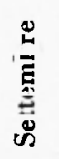 & 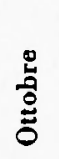 & 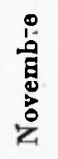 & $\begin{array}{l}\text { 苞 } \\
\text { 苞 } \\
.0\end{array}$ \\
\hline & & & & & & & & & & & & \\
\hline 2 & $\overline{10}$ & - & - & - & - & - & 1.7 & 0.4 & 0.4 & 0.1 & - & - \\
\hline 3 & - & - & - & - & - & 0.4 & 1.3 & - & 1.1 & - & - & - \\
\hline 4 & 1.2 & - & - & - & - & 0.2 & 1.2 & 0.5 & 1.2 & - & - & - \\
\hline 5 & 1.6 & - & - & - & 0.3 & 0.1 & 0.1 & 1.3 & - & - & - & - \\
\hline 6 & - & - & - & - & - & - & - & 1.4 & - & - & - & - \\
\hline 7 & 1.1 & 0.1 & - & - & - & - & 0.6 & - & - & - & - & - \\
\hline 8 & 0.0 & - & - & 0.3 & 0.9 & - & - & - & - & - & 0.6 & - \\
\hline 9 & - & 1.6 & - & 0.5 & 0.6 & 0.4 & - & 1.2 & 1.2 & 0.6 & - & - \\
\hline 10 & - & - & - & 0.5 & 0.2 & - & 0.3 & - & - & - & - & 0.1 \\
\hline 11 & - & - & 0.8 & - & - & 0.4 & 0.6 & 1.4 & - & - & - & - \\
\hline 12 & - & - & - & - & - & - & 1.0 & 1.2 & - & - & - & - \\
\hline 13 & - & - & - & - & 0.3 & 0.1 & - & 1.2 & - & - & 0.1 & - \\
\hline 14 & - & - & 1.4 & $\bar{q}$ & - & - & 0.2 & - & - & - & - & - \\
\hline 15 & 2.0 & - & $1 . \overline{5}$ & 0.7 & 0.7 & 一 & 0.4 & - & - & - & 0.1 & - \\
\hline 16 & - & - & - & - & - & - & 0.8 & - & - & - & - & 0.6 \\
\hline 17 & - & - & - & 0.8 & - & 0.2 & 1.7 & - & - & 0.3 & - & - \\
\hline 18 & - & 2.0 & 0.2 & 0.8 & - & - & - & - & - & 0.6 & - & - \\
\hline 19 & 2.6 & 1.5 & - & 1.1 & - & - & 1.9 & 一 & - & - & - & 0.3 \\
\hline 20 & - & 0.6 & - & - & - & - & - & - & - & - & 1.2 & - \\
\hline 21 & 2.4 & - & 0.1 & - & 0.2 & - & 1.9 & - & - & - & 0.8 & - \\
\hline 22 & - & 一 & 0.1 & - & - & - & 1.7 & 1.0 & - & - & - & - \\
\hline 23 & - & - & - & - & 0.2 & - & 1.6 & - & 0.6 & - & - & 0.2 \\
\hline 24 & 一 & - & 一 & - & 0.3 & - & 1.4 & - & 0.8 & - & - & - \\
\hline 25 & - & 2.4 & - & - & - & - & - & - & - & - & - & - \\
\hline 26 & - & 2.4 & - & 0.9 & - & - & 0.7 & $1 . \mathrm{L}$ & 0.2 & - & - & - \\
\hline 27 & - & 2.8 & 0.0 & - & - & - & - & 0.9 & - & - & - & - \\
\hline 28 & 0.7 & - & - & - & - & 0.8 & $\therefore$ & 0.5 & - & 0.7 & - & - \\
\hline 29 & 0.6 & - & - & 0.5 & - & - & 0.7 & 0.0 & - & - & - & - \\
\hline 80 & - & - & - & - & 0.7 & 1.6 & 0.8 & - & - & - & - & - \\
\hline 31 & - & - & - & - & 1.2 & & 0.7 & - & & & & - \\
\hline Media & 1.3 & 1.6 & 0.6 & 0.7 & 0.4 & 0.5 & 1.0 & ก.9 & 0.7 & 0.5 & 0.6 & 0.3 \\
\hline
\end{tabular}

